'Departamento de Dermatología. Departamento de Anatomía Patológica. Facultad de Medicina, Pontificia Universidad Católica, Santiago de Chile.

aInterna de Medicina Facultad de Medicina, Pontificia Universidad Católica, Santiago de Chile.

Los autores declaran ausencia de conflictos de intereses.

Recibido el 5 de septiembre de 2013, aceptado el 11 de noviembre de 2013

Correspondencia: Dra. Montserrat Molgó N Dermatología, Centro Médico San Joaquín.

Av. Vicuña Mackenna 4686 Macul, Santiago, Chile. Teléfono: (56 2) 23548659 Fax: (56 2) 25529974 montsemolgo1@gmail.com

\section{Necrosis cutánea: un desafío para el médico}

\author{
MONTSERRAT MOLGÓ N. ${ }^{1}$, CAMILA ARRIAGADA E.. , \\ CLAUDIA SALOMONE B. ${ }^{1}$, CRISTIÁN VERA K. ${ }^{1}$, LAURA GIESEN F. ${ }^{1}$, \\ ANTONIETA SOLAR G. ${ }^{2}$, SERGIO GONZÁLEZ B. ${ }^{2}$
}

\section{Skin necrosis. Report of eleven cases}

Skin necrosis must be considered as a syndrome, because it is a clinical manifestation of different diseases. An early diagnosis is very important to choose the appropriate treatment. Therefore, its causes should be suspected and confirmed quickly. We report eleven patients with skin necrosis seen at our Department, caused by different etiologies: Warfarin-induced skin necrosis, loxoscelism, diabetic microangiopathy, ecthyma gangrenosum, disseminated intravascular coagulation, necrotizing vasculitis, paraneoplastic extensive necrotizing vasculitis, livedoid vasculopathy, necrotizing fasciitis, necrosis secondary to the use of vasoactive drugs and necrosis secondary to the use of cocaine. We also report the results of our literature review on the subject.

(Rev Med Chile 2014; 142: 118-124)

Key words: Necrobiotic disorders; Skin diseases; Vasculitis.
L a necrosis cutánea debe ser considerada como un síndrome, pues es la manifestación cutánea de diversas entidades clínicas. El diagnóstico precoz y la pronta identificación de la etiología son claves para un tratamiento oportuno y mejorar la sobrevida del paciente.

Se recolectaron los casos más demostrativos de necrosis cutánea en pacientes hospitalizados. Se registró la historia clínica, se tomaron fotografías y en algunos casos se realizaron biopsias de las lesiones.

\section{Casos clínicos}

En la Tabla 1 se presentan los casos clínicos más demostrativos de necrosis cutánea en pacientes hospitalizados en nuestra institución. Se detalla sexo del paciente, edad, antecedentes mórbidos, fármacos utilizados, clínica con la que se presentaron al momento de la consulta y el estudio de anatomía patológica (AP). Finalmente, se describe el diagnóstico y se menciona el tratamiento que se realizó.

\section{Caso 1: Necrosis por cumarínicos}

Se ha descrito necrosis cutánea asociada al uso de warfarina, reportándose una prevalencia entre 0,01 y $0,1 \%$ de los pacientes tratados. Aunque el mecanismo no está aclarado, se describe un efecto procoagulante transitorio por una rápida caída de los niveles de proteína $\mathrm{C}^{1,2}$. El cuadro clínico se manifiesta como edema y eritema de inicio súbito, asociado a dolor o parestesias. En $90 \%$ de los casos, la aparición de necrosis cutánea se produce de 3 a 6 días de iniciado el tratamiento, aunque se han descrito casos que se han desarrollado semanas o meses después ${ }^{3}$. El estudio histopatológico revela trombosis y depósitos de fibrina en las vénulas postcapilares sin inflamación $\mathrm{n}^{4,5}$. El tratamiento es la suspensión del fármaco, transfusión de plasma fresco congelado y aporte de vitamina $\mathrm{K}$ endovenosa.

\section{Caso 2: Loxoscelismo cutáneo necrótico}

El loxoscelismo es el cuadro clínico secundario a la mordedura de araña del género Loxosceles laeta, el cual presenta una forma limitada a la piel y otra sistémica (loxoscelismo cutáneo visceral), la que 


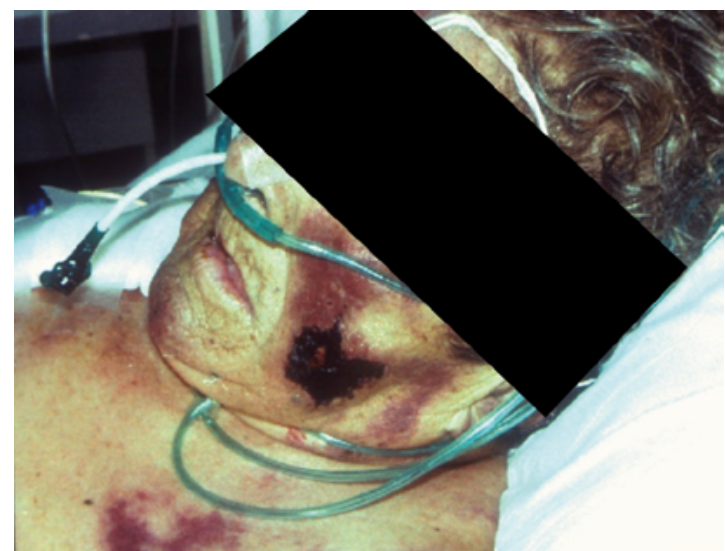

Figura 1. Equímosis y zonas de necrosis en cara por ectima gangrenoso.

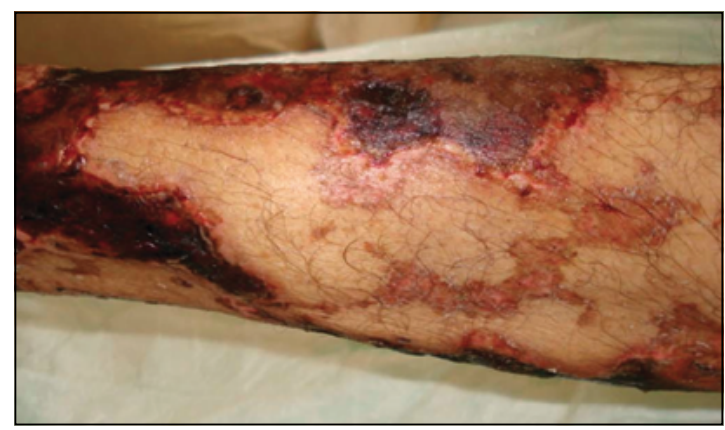

Figura 3. Lesiones eritemato-violáceas y necrosis extensa por vasculitis necrotizante sistémica extensa paraneoplásica.

se caracteriza por anemia hemolítica, hematuria, ictericia, fiebre, compromiso de conciencia e insuficiencia renal aguda, con una mortalidad cercana a $20 \%{ }^{6}$. El loxoscelismo cutáneo necrótico presenta la placa livedoide dentro de las primeras $24 \mathrm{~h}$, producto de una vasculitis secundaria a la mordedura e inoculación del veneno ${ }^{7}$. Es una placa violácea dolorosa, rodeada por un halo eritematoso. Evoluciona a la formación de una escara necrótica que se desprende y deja una úlcera que cicatriza lentamente ${ }^{8}$. El tratamiento consiste en aplicación de hielo local, elevación de la extremidad, corticoides sistémicos, analgesia y antihistamínicos. Puede usarse el suero anti-loxosceles que debe administrarse dentro de las primeras $4 \mathrm{~h}$ de ocurrida la mordedura ${ }^{9}$.

\section{Caso 3: Microangiopatía diabética}

La isquemia en las extremidades inferiores es una complicación a largo plazo de la diabetes. Los

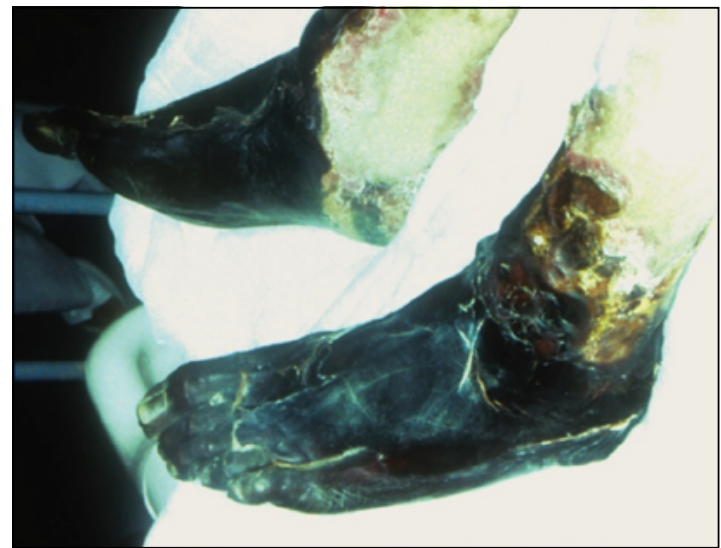

Figura 2. Extensas placas necróticas secas en ambos pies por CID.

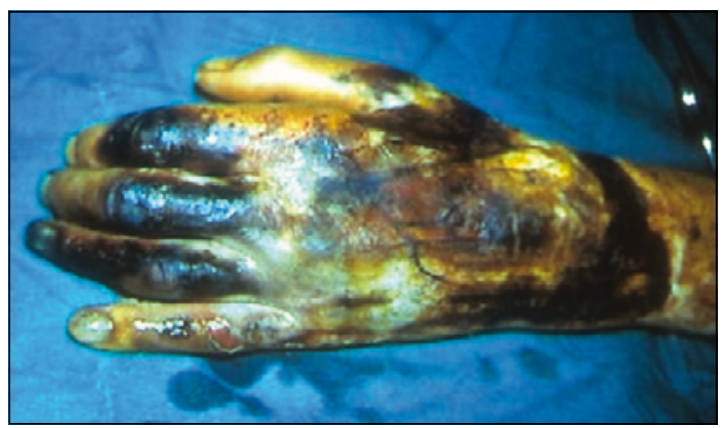

Figura 4. Necrosis de mano por fasceítis necrotizante.

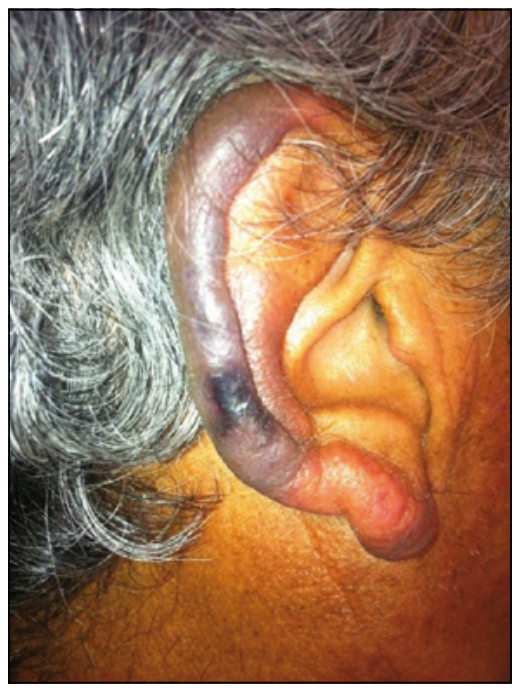

Figura 5. Cianosis y necrosis de pabellón auricular por necrosis por cocaína. 


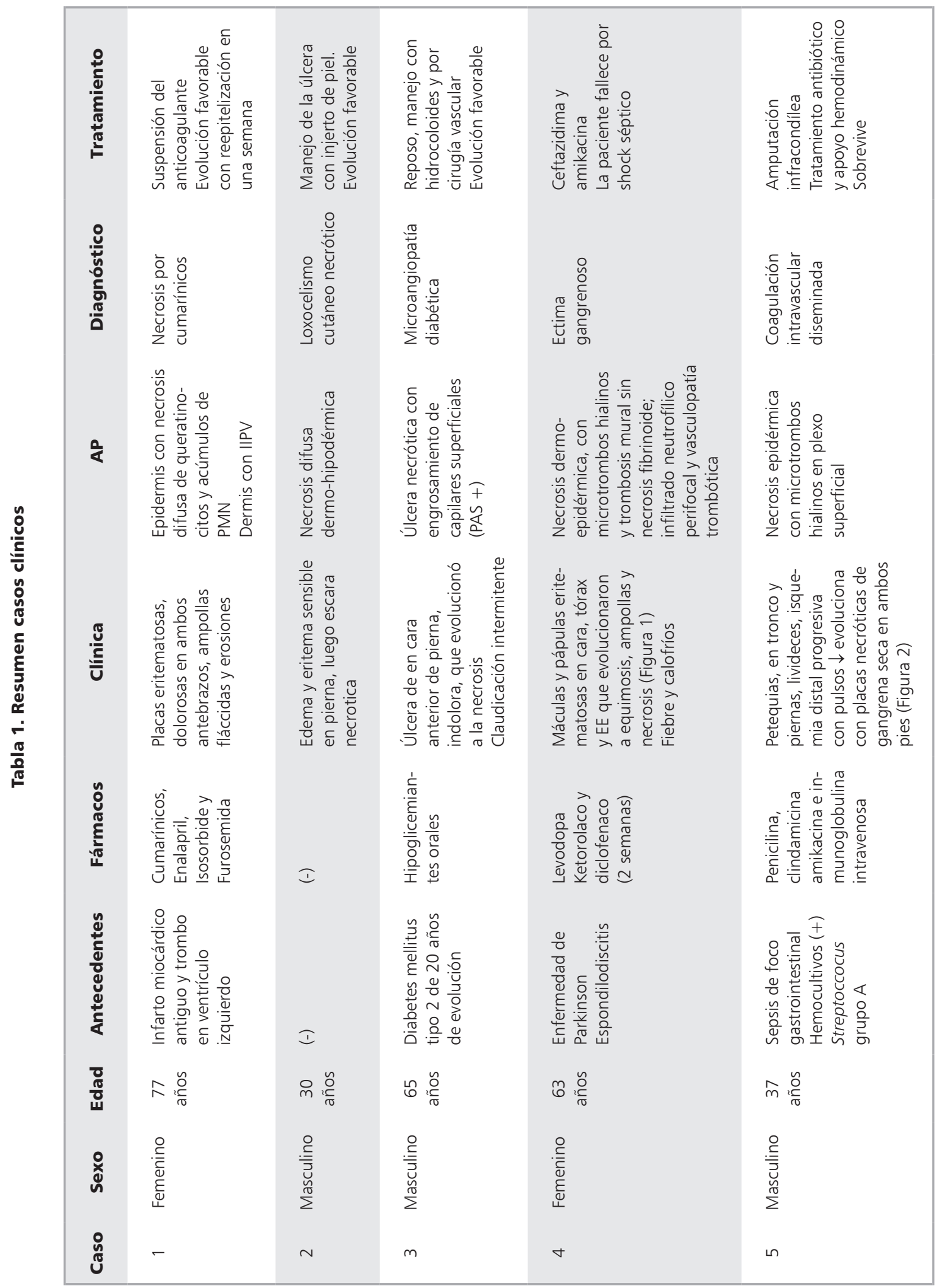




\begin{tabular}{|c|c|c|c|c|c|}
\hline 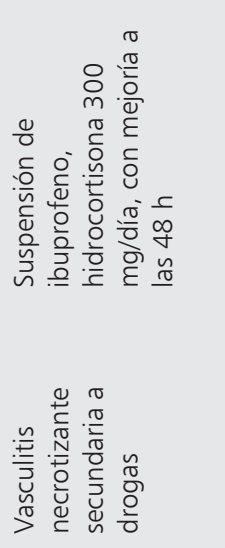 & 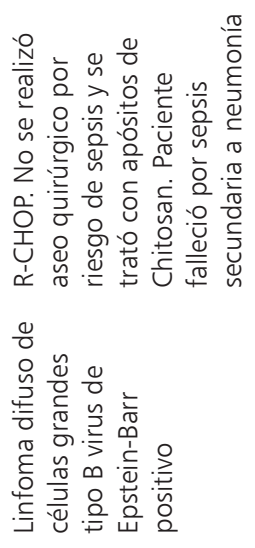 & 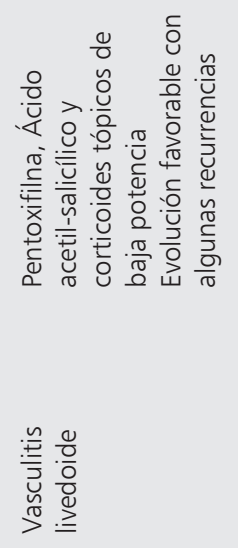 & 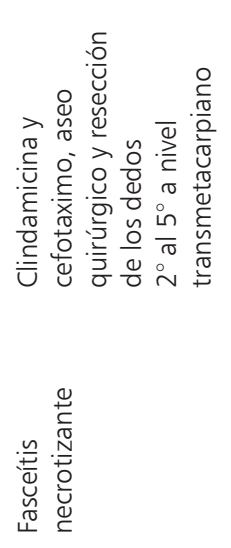 & 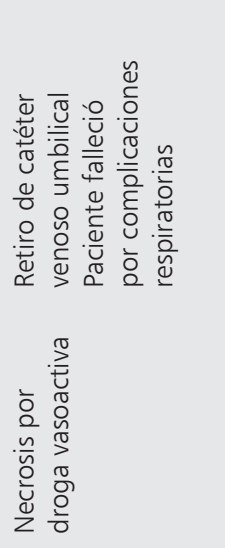 & 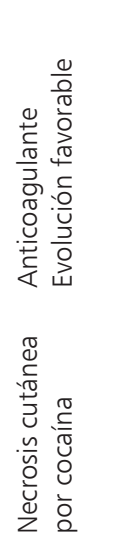 \\
\hline 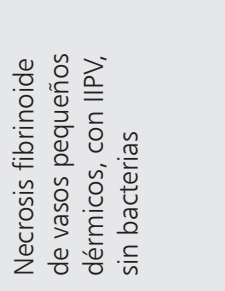 & 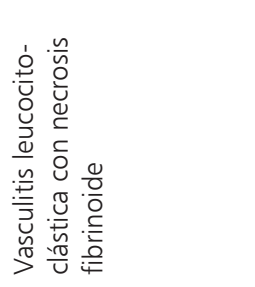 & 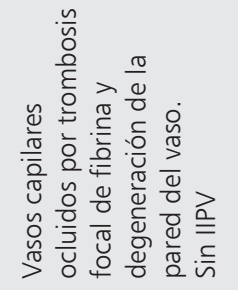 & 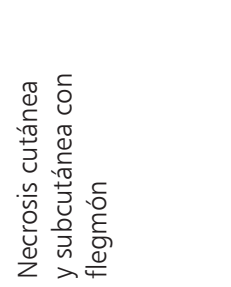 & 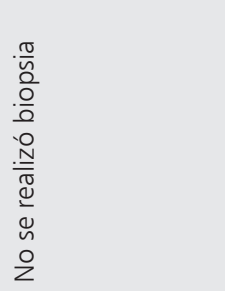 & 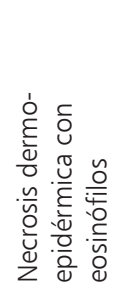 \\
\hline 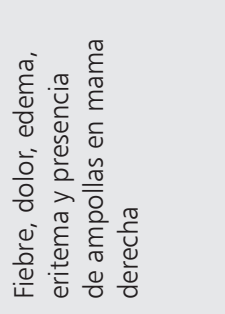 & 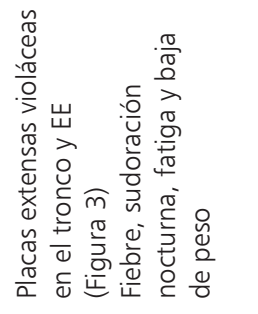 & 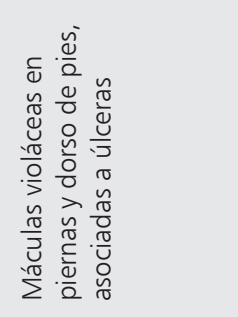 & 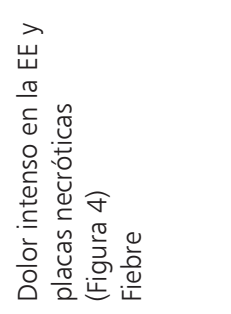 & 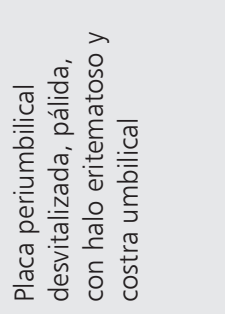 & 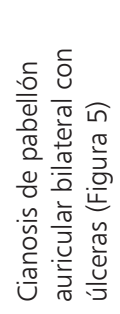 \\
\hline 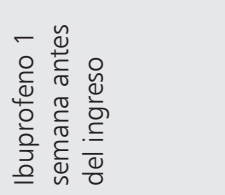 & 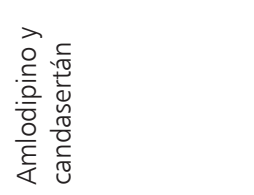 & ' & 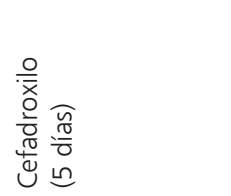 & 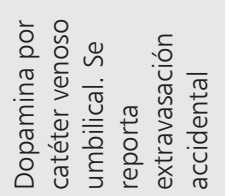 & 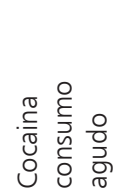 \\
\hline 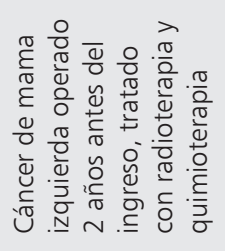 & 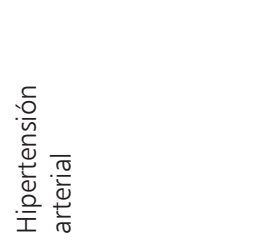 & ' & 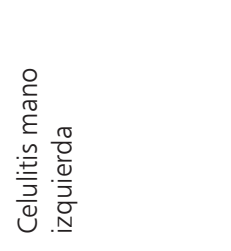 & 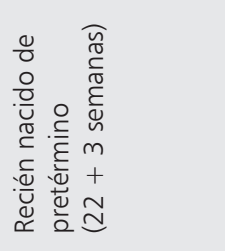 & 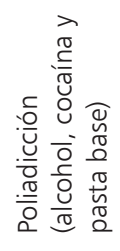 \\
\hline 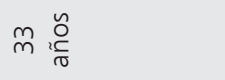 & 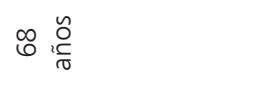 & 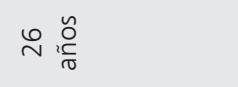 & 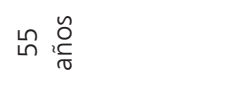 & $\stackrel{c}{\stackrel{c}{0}}$ & กิ \\
\hline 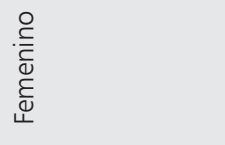 & 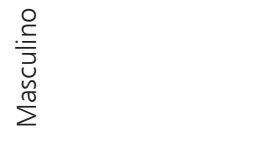 & 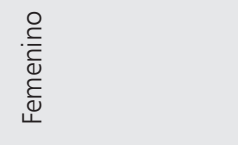 & 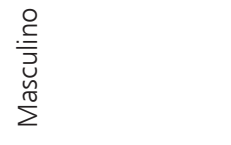 & 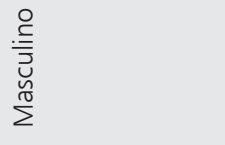 & 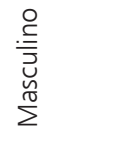 \\
\hline 6 & $\wedge$ & $\infty$ & $\sigma$ & $\stackrel{\circ}{\circ}$ & $\mp$ \\
\hline
\end{tabular}


pacientes evolucionan con claudicación intermitente $y$, posteriormente, con dolor de reposo asociado a alteraciones de la sensibilidad ${ }^{10,11}$. Pueden presentar alteraciones tróficas de las extremidades, engrosamiento de las uñas, xerosis, edema, linfangitis, pulsos disminuidos, piel fría, eritema, palidez o cianosis. Finalmente, aparecen úlceras y necrosis ${ }^{12,13}$. La ecografía Doppler y la angiografía permiten cuantificar la magnitud del deterioro de la circulación arterial. El tratamiento incluye ejercicio físico, manejo de la diabetes y de los otros factores de riesgo ${ }^{14}$. El tratamiento definitivo es la revascularización ${ }^{15}$.

\section{Caso 4: Ectima gangrenoso}

El ectima gangrenoso es una manifestación cutánea de la infección por pseudomonas, por diseminación hematógena (forma clásica o bacterémica) o inoculación local del microorganismo ${ }^{16}$. Las lesiones se inician como placas eritematosas, dolorosas, que desarrollan una bula hemorrágica central que se necrosa ${ }^{17}$. Puede asociarse a vasculitis sistémica y crioglobulinemia, cuyos síntomas pueden iniciarse después del inicio del tratamiento ${ }^{18}$. El estudio histológico muestra necrosis dermo-epidérmica y, en ocasiones, bacterias invaden los vasos sanguíneos, con signos de vasculitis necrotizante, pero con escasas células inflamatorias. El tratamiento consiste en el inicio precoz de antibióticos endovenosos según antibiograma ${ }^{19}$.

\section{Caso 5: Coagulación intravascular diseminada (CID)}

La CID se asocia a infecciones severas, tumores, falla hepática, enfermedades inmunológicas, traumatismos con gran daño tisular, alteraciones vasculares y patologías obstétricas ${ }^{20,21}$. Las manifestaciones clínicas más frecuentes son hemorragias de piel y mucosas, con púrpura y petequias, bulas hemorrágicas y hematomas subcutáneos. En los exámenes destacan prolongación del TP y TTPA, fibrinógeno disminuido, aumento del dímero D, trombocitopenia y fragmentación de glóbulos rojos. El tratamiento es el de la enfermedad de base, además de apoyo circulatorio, ventilación y hemodiálisis $^{22}$. Si el paciente presenta sangrado, deben indicarse transfusión de plaquetas, plasma fresco congelado o crioprecipitados. En pacientes con trombosis como manifestación principal, se debe iniciar de inmediato terapia con heparina endovenosa ${ }^{20}$.

\section{Casos 6 y 7: Vasculitis necrotizante y vasculitis necrotizante sistémica extensa paraneoplásica}

La vasculitis con compromiso cutáneo puede ser idiopática (45-55\%), secundaria a infección (15-20\%), a enfermedad inflamatoria (15-20\%), a drogas (10-15\%) o a neoplasias malignas $(<5 \%)$. La vasculitis leucocitoclástica (VL) es una vasculitis de vaso pequeño y es mediada por depósito de complejos inmunes (IgG e IgM), en los vasos postcapilares. Esto activa la cascada del complemento y se liberan factores quimiotácticos y enzimas lisosomales que producen la destrucción de la pared del vaso sanguíneo, dando lugar al púrpura palpable que evoluciona a pápulas, vesículas hemorrágicas, úlceras y lesiones necróticas ${ }^{23}$. El tratamiento incluye el manejo de las causas subyacentes y corticoides sistémicos por 10 a 14 días $^{24}$. En el caso 6 se presenta una vasculitis necrotizante con biopsia compatible con VL probablemente secundaria a drogas. En el caso 7 se presenta una vasculitis necrotizante sistémica extensa asociada a linfoma difuso de células grandes tipo B del anciano asociado a virus Epstein Barr, en el que la vasculitis necrotizante probablemente representa una manifestación paraneoplásica ${ }^{25}$.

\section{Caso 8: Vasculitis livedoide}

La vasculitis livedoide es una enfermedad crónica caracterizada por un trastorno oclusivo de la microcirculación. La manifestación clínica es una tríada que consiste en distribución reticulada de máculas y pápulas purpúricas, úlceras episódicas dolorosas y curación con cicatriz atrófica blanquecina conocida como atrofia blanca. Existen 2 tipos: idiopática y secundaria (enfermedad del tejido conectivo). Frecuentemente se asocia a coagulación alterada y se pueden encontrar marcadores protrombóticos como activación de plaquetas y un estado de hipercoagulabilidad primario o secundario $^{26}$. En la histopatología, se observan vasos capilares ocluidos por trombosis focal de fibrina y degeneración de la pared del vaso. A diferencia de la vasculitis inflamatoria primaria, existe mínimo infiltrado inflamatorio perivascular. El tratamiento incluye antiagregantes plaquetarios y anticoagulación sistémica, de preferencia heparina de bajo peso molecular ${ }^{26}$.

\section{Caso 9: Fascitis necrotizante}

La fascitis necrotizante es una infección del tejido subcutáneo que puede ser producida por el 
estreptococo $\beta$-hemolítico grupo A (tipo I) o ser polimicrobiana (tipo II), con bacterias aeróbicas y anaeróbicas ${ }^{27}$. Se presenta como una celulitis con dolor desproporcionado, que evoluciona a una placa indurada con lesiones bulosas o purpúricas. Hay signos de toxicidad sistémica hasta falla orgánica múltiple ${ }^{28}$. La biopsia incisional del centro de la lesión puede ayudar al diagnóstico. En el estudio histopatológico hay necrosis epidérmica, edema y hemorragia dérmica focal, trombosis vascular prominente, infiltrado de neutrófilos y numerosos diplococos gram positivos ${ }^{29}$. El tratamiento incluye antibióticos y debridamiento quirúrgico del tejido necrótico, el que iniciado en forma precoz mejora el pronóstico del paciente ${ }^{30}$.

\section{Caso 10: Necrosis por droga vasoactiva}

La reacción cutánea severa secundaria a dopamina ocurre cuando existe extravasación en tejidos perivasculares, lo que produce vasoconstricción a través de su efecto estimulante alfa-adrenérgico. Cuando ocurre extravasación y el tejido adyacente está desvitalizado, se puede administrar fentolamina que es un bloqueador alfa-adrenérgico ${ }^{31}$. En nuestro paciente no se utilizó, por el tiempo transcurrido y la buena evolución espontánea ${ }^{32}$. Es importante considerar los diagnósticos diferenciales que, si no se sospechan y tratan, pueden ocasionar muerte del paciente. Dentro de estos diagnósticos es necesario considerar las complicaciones infecciosas del cordón umbilical.

\section{Caso 11: Necrosis por cocaína}

Se ha descrito necrosis de segmentos distales secundario al abuso de cocaína. Se asocia principalmente al uso de droga endovenosa, sin embargo, también se ha descrito después de aspiración. La cocaína es un potente agente vasoconstrictor ya que bloquea la recaptura de norepinefrina, resultando en el aumento de la estimulación alfa adrenérgica, aumento en la producción de endotelina 1 y disminución de la liberación de óxido nítrico en las células endoteliales. Esto genera un prolongado y repetido vasoespasmo que favorece la isquemia cutánea e infartos ${ }^{33}$. El tratamiento incluye terapia anticoagulante y antitrombótica, incluso pudiendo requerir fasciotomía de emergencia y amputación. Como terapia adicional se debe considerar el uso de vasodilatadores intravenosos.

\section{Comentario final}

Del análisis de las diversas condiciones causantes de necrosis cutáneas puede concluirse que es muy importante que el médico, al enfrentarse a un síndrome de necrosis cutánea, piense e identifique las causas que pueden ocasionar esta manifestación clínica similar, ya que el inicio de una terapia adecuada y oportuna evitará complicaciones y favorecerá el pronóstico del paciente. La mayoría de estas condiciones requieren hospitalización en unidades de mayor complejidad, ya que pueden evolucionar rápidamente con falla multiorgánica. Es de especial importancia el rol del dermatólogo y del dermatopatólogo, ya que la biopsia cutánea puede ser fundamental para determinar con precocidad el diagnóstico.

Agradecimientos: Agradecemos al Dr. Jorge Vergara del Departamento de Traumatología de la Pontificia Universidad Católica de Chile por aportarnos con casos clínicos.

\section{Referencias}

1. Chan YC, Valenti D, Mansfield AO, Stansby G. Warfarin induced skin necrosis. Br J Surg 2000; 87: 266-72.

2. Miyamotto M, Rocha R, Fretas J, Erzinger F, Lagos H. Necrose de mama relacionada ao uso de warfarina. J Vasc Br 2004; 3: 52-4.

3. Kaiber FL, Malucelli TO, Baroni ERV, Schafranski MD, Akamatsu HT, Schmidt CCF. Heparin-induced thrombocytopenia and warfarin-induced skin necrosis: a case report. An Bras Dermatol 2010; 85: 918-8.

4. Essex DW, Wynn SS, Jin DK. Late-onset warfarininduced skin necrosis: case report and review of the literature. Am J Hematol 1998; 57: 233-7.

5. Scarff CE, Baker C, Hill P, Foley P. Late-onset warfarin necrosis. Australas J Dermatol 2002; 43: 202-6.

6. Schenone H. Cuadros tóxicos producidos por mordeduras de araña en Chile. lactrodectismo y loxocelismo. Rev Med Chile 2003; 131: 437-44.

7. Sánchez-Olivas MA, Valencia-Zavala MP, Sánchez-Olivas JA, Sepúlveda-Velázquez, Vega-Robledo G. Loxoscelismo cutáneo necrótico. Informe de un caso. Rev Alergia Mex 2011; 58: 171-6.

8. Elston DM, Miller SD, Young RJ 3rd, Eggers J, McGlasson $\mathrm{D}$, Schmidt WH, et al. Comparison of colchicine, dapsone, triamcinolone, and diphenhydramine therapy for the treatment of brown recluse spider envenomation: 
a double-blind, controlled study in a rabbit model. Arch Dermatol 2005; 141: 595.

9. Sams H. Necrotic arachnidism. J Am Acad Dermatol 2001; 44: 561-73.

10. España G. Capítulo 12. Enfermedad vascular periférica: isquemia de miembros Inferiores. En Estudio de la incapacidad laboral por enfermedades cardiovasculatorias. U. Sosa Rodríguez. Instituto Nacional de Medicina y Seguridad del Trabajo Madrid 1998. Pág. 161-171. Disponible en internet.

11. Santos D, Carline T. Examination of the lower limb in high risk patients. J Tissue Viability 2000; 10: 97-105.

12. Ghirlanda G, Citterio F. Lower limb ischemia. Rays 1997; 22: 535-43.

13. Creager M, Dzau V. Enfermedades vasculares de las extremidades. En: Harrison's. Principios de Medicina Interna. Capítulo 248. 14ª Edición. 1998. Editado por Anthony Fauci, et al. Vol. I, pag. 1593-602.

14. Camera A, Hopps E, Caimi G. Diabetic microangiopathy: physiopathological, clinical and therapeutic aspects. Minerva Endocrinol 2007; 32: 209-29.

15. Schindl A, Schindl M, Schon H, Knobler R, Havelec L, Schindl L. Low-intensity laser irradiation improves skin circulation in patients with diabetic microangiopathy. Diabetes Care 1998; 21: 580-4.

16. Huminer D, Siegman-Igra Y, Morduchowicz G, Pitilik SD. Ecthyma gangrenosum without bacteremia. Arch Intern Med 1987; 147: 299-301.

17. Greene SL, Su WPD, Muller SA. Ecthyma gangrenosum: report of clinical, histopathologic and bacteriologic aspects of eight cases. J Am Acad Dermatol 1984; 11: 781-7.

18. Zomorrodi A, Wald ER. Ecthyma gangrenosum: considerations in a previously healthy child. Pediatr Infect Dis J 2002; 21: 1161-4.

19. Puzenat E, Chirouze C, Khayat N, Aubin F, Estavoyer JM, Humbert P, et al. Ecthyma gangrenosum caused by Pseudomonas stutzeri with bacteraemia and systemic vascularitis. Rev Med Interne 2004; 25: 315-8.

20. Hock C. Disseminated intravascular coagulation: old disease, new hope. Br Med J 2003; 327: 974-7.

21. Bakhshi S. Diagnosis and treatment of disseminated intravascular coagulation. Indian Pediatr 2003; 40: 721-30.

22. Páramo J. Coagulación intravascular diseminada. Med Clin (Barc). 2006; 127: 785-9.

23. Arvan M, Brodell R. A palpable clue to vasculitis. Postgraduate Medicine On-line 1999; 105 (3).

24. Fiorentino D. Cutaneous vasculitis. J Am Acad Dermatol 2003; 48: 311-40.

25. Zoroquiain P, González S, Molgó M, Rodríguez A, Valbuena JR. Leukocytoclastic Vasculitis as Early Manifestation of Epstein-Barr Virus-Positive Diffuse Large B-Cell Lymphoma of the Elderly. Am J Dermopathol 2012; 34: 330-4.

26. Kerk N, Goerge T. Livedoid vasculopathy - current aspects of diagnosis and treatment of cutaneous infarction. J Dtsch Dermatol Ges 2013; 11: 407-10.

27. Brun-Buisson C. The therapeutic approach to necrotizing fasciitis. Ann Dermatol Venereol 2001; 128: 394403.

28. Aronoff DM, Bloch KC. Assessing the relationship between the use of nonsteroidal antiinflammatory drugs and necrotizing fasciitis caused by group A streptococcus. Medicine (Baltimore) 2003; 82: 225-35.

29. Dahl PR, Perniciario C, Holmkvist KA, O’Connor MI, Gibson LE. Fulminant group A streptococcal necrotizing fasciitis: Clinical and pathologic findings in 7 patients. J Am Acad Dermatol 2002; 47: 489-92.

30. Howie WO. Anesthetic implications of necrotizing fasceitis. AANA J 2003; 71: 37-40.

31. Subhani M, Sridhar S, DeCristofaro JD. Phentolamine use in a neonate for the prevention of dermal necrosis caused by dopamine: a case report. J Perinatol 2001; 21: 324-6.

32. Molgo M, Naranjo M. Necrosis periumbilical secundaria a droga vasoactiva. Rev chilena Dermatología 2006; 22: 117-9.

33. Del Giudice P. Cutaneous complications of intravenous drug abuse. Br J Dermatol 2004; 150: 1-10. 